\title{
Incremental Learning of Planning Actions in Model-Based Reinforcement Learning
}

\author{
Jun Hao Alvin Ng ${ }^{1,2}$ and Ronald P. A. Petrick ${ }^{1}$ \\ ${ }^{1}$ Department of Computer Science, Heriot-Watt University \\ ${ }^{2}$ School of Informatics, University of Edinburgh \\ alvin.ng@ed.ac.uk, R.Petrick@hw.ac.uk
}

\begin{abstract}
The soundness and optimality of a plan depends on the correctness of the domain model. Specifying complete domain models can be difficult when interactions between an agent and its environment are complex. We propose a model-based reinforcement learning (MBRL) approach to solve planning problems with unknown models. The model is learned incrementally over episodes using only experiences from the current episode which suits non-stationary environments. We introduce the novel concept of reliability as an intrinsic motivation for MBRL, and a method to learn from failure to prevent repeated instances of similar failures. Our motivation is to improve the learning efficiency and goaldirectedness of MBRL. We evaluate our work with experimental results for three planning domains.
\end{abstract}

\section{Introduction}

Planning requires as input a model which describes the dynamics of a domain. While domain models are normally hand-coded by human experts, complex dynamics typical of real-world applications can be difficult to capture in this way. This is known as the knowledge engineering problem [Cullen and Bryman, 1988]. One solution is to learn the model from data which is then used to synthesize a plan or policy. In this work, we are interested in applications where the training data has to be acquired by acting or executing an action. However, training data acquired in an episode could be insufficient to infer a complete model. While this is mitigated by including past training data from previous episodes, this would be ill-suited for non-stationary domains where distributions of stochastic dynamics shift over time.

Following these observations, we present the incremental learning model (ILM), a model-based reinforcement learning (MBRL) approach, which learns and refines action models incrementally over episodes and uses the learned model to synthesize a plan or policy. We use MBRL as it could be more sample-efficient than model-free approaches and the learned models can be reused to achieve different tasks. Relational rules are used to represent learned models (see Figure 1). Previously learned action models, or prior action models, are provided to subsequent episodes and are improved upon acquiring new training data; past training data is not used. Prior action models could also be hand-coded incomplete models serving as prior knowledge. Our training data consists of state transitions $\left(s_{t}, a_{t}, s_{t+1}\right)$ where $s_{t}$ is the pre-state, $a_{t}$ is the grounded action, and $s_{t+1}$ is the post-state.

While the learning progress cannot be determined without the true action models, we can estimate it empirically based on the results of learning and acting. This empirical estimate, or reliability, is used to guide the search in the space of possible models during learning and as an intrinsic motivation in reinforcement learning. When every action is sufficiently reliable, we instead exploit with Gourmand [Kolobov et al., 2012], a planner that solves finite-horizon Markov Decision Processes (MDP) problems online. The learned rules are translated to PPDDL, a planning language modelling probabilistic planning problems [Younes and Littman, 2004], and given as inputs to the planner.

Another major contribution of our work is its ability to learn from failure. Actions fail to be executed if their preconditions are not satisfied in the current state. This is common when the model is incorrect. Failed executions can have dire consequences in the real-world or cause irreversible changes such that goal states cannot be reached. ILM records failed executions and prevents any further attempts that would lead to similar failures. This reduces the number of failed executions and increases the efficiency of exploration.

The rest of the paper is organized as follows. First, we review related work and present the necessary background. Next, we provide details of ILM. Lastly, we evaluate ILM in three planning domains and discuss the significance of our contributions. Results show that even without past training data, the models can be improved incrementally.

\section{Related Work}

R-MAX [Brafman and Tennenholtz, 2002] is a provably sample-efficient MBRL algorithm which handles the balance between exploration and exploitation implicitly by assigning the maximum reward to unknown states which are set to absorbing states. If the count, defined as the number of times an action is executed in the state, of every applicable action exceeds a threshold, then the state is known. R-MAX is impractical for planning problems with large state spaces. Hence, additional assumptions such as factored state spaces [Kearns 
Name: moveCar(?from ?to)

Precondition: at(?from) $\wedge$ road(?from ?to) $\wedge$ notFlattire()

Effect: 0.75 at(?to) $\wedge \neg$ at(?from)

0.25 at(?to) $\wedge \neg$ at (?from) $\wedge \neg$ notFlattire ()

$0\langle$ noise $\rangle$

Figure 1: The rule for the true action model representing moveCar in the Tireworld domain with arguments ?from and ?to.

and Koller, 1999], known structures of dynamic Bayesian networks (DBN) [Guestrin et al., 2002], or known maximum indegree of DBNs [Diuk et al., 2009] are often made. Conversely, we only assume that the arguments of actions are known. We also use an empirical estimate for the learning progress, which we call reliability, as intrinsic motivation. Reliability is also used to quantify prior knowledge which other works on intrinsic motivation do not address [Chentanez et al., 2005; Hester and Stone, 2017].

We extend the rules learner from [Pasula et al., 2007] to learn action models. A set of relational rules represent an action which can have probabilistic effects. A relational representation allows generalization of the state space unlike propositional rules which are used in [Oates and Cohen, 1996]. [Mourão et al., 2012; Martínez et al., 2016] learn probabilistic actions but do not address the incremental nature of reinforcement learning. [Gil, 1994; Wang, 1995] learn deterministic action models incrementally while [Rodrigues et al., 2010] learns probabilistic action models. Our work is most similar to the latter which revises relational rules whenever contradicting examples are received. They do not store all the examples but rather track how well each rule explains the examples. On the other hand, we address incremental learning over episodes where past training data is not used. Furthermore, our approach could consider prior knowledge in the form of incomplete action models which can have extraneous predicates unlike [Zhuo et al., 2013].

\section{Background}

PPDDL. Action models described in PPDDL are defined by their preconditions and effects, typically restricted to conjunctions of predicates. An action is applicable if its preconditions are true in the current state. Executing an applicable action changes the state according to its effects which can be deterministic or probabilistic.

Rules. For learning action models, we use a rule-based representation as it is well-suited to the incremental nature of reinforcement learning [Rodrigues et al., 2010]. An action is described by a set of rules $R$ where a rule $r \in R$ has three parts: the name of the action, the precondition, and the effect. An example is shown in Figure 1. The noise effect serves to avoid modelling a multitude of rare effects which could increase the complexity of synthesizing a plan. When a rare effect occurs, it is often better to replan. If the action has disjunctive preconditions or effects, then multiple rules are required to represent it. A rule covers a state-action pair $(s, a)$ if it represents $a$ and is applicable in $s$. Every stateaction pair in the training data is covered by at most one rule which is called the unique covering rule, denoted as $r_{(s, a)}$. A propositional rule is obtained from the grounding of a relational rule by assigning an object or value to every argument in the rule (e.g., grounding moveCar(?loc1, ?loc2) to moveCar(l31, l13)). Actions are grounded in a similar fashion.

Markov Decision Processes (MDPs). MDPs model fullyobservable problems with uncertainty. A finite-horizon MDP is a tuple of the form $\left(\mathcal{S}, \mathcal{A}, T, R, \mathcal{G}, s_{0}, H\right)$ where $\mathcal{S}$ is a set of states, $\mathcal{A}$ is the set of actions, $T: \mathcal{S} \times \mathcal{A} \times \mathcal{S} \rightarrow[0,1]$ is the transition function, $R: \mathcal{S} \times \mathcal{A} \rightarrow \mathbb{R}$ specifies rewards for performing actions, $\mathcal{G}$ is the set of goal states, $s_{0}$ is the initial state, and $H$ is the number of decision epochs or planning horizon. The objective is to find a policy which maximizes the sum of expected rewards.

Reinforcement Learning. When transition functions in MDPs are not known, MBRL can be used to learn them and perform sequential decision-making. This is the same as learning action models as they can be translated to transition functions [Younes and Littman, 2004]. Reinforcement learning deals with the balance between exploration and exploitation. Exploration seeks meaningful experiences from which action models are learned while exploitation synthesizes a policy using the models.

\section{Incremental Learning Model}

We first introduce the concept of reliability which is used as intrinsic motivation in MBRL and in the rules learner. This is followed by an outline of the main I LM algorithm. Lastly, we provide details of the extension made to the rules learner from [Pasula et al., 2007].

\subsection{Reliability of Actions}

The reliability of learned action models are empirical estimates of its learning progress. Reliability serves two purposes. We extend the rules learner to consider the prior action model and its reliability to learn new rules. In reinforcement learning, less reliable actions are preferred during exploration. Reliability is defined as:

$$
R E(o)=E X(o)\left(\alpha_{s} S U(o)-\alpha_{v} V O(o)\right)+\beta^{n} R E\left(o_{0}\right)
$$

where $o$ is an action, $E X$ is the exposure, $S U$ is the success rate, $V O$ is the volatility, $\alpha_{s}, \alpha_{v}$, and $\beta$ are scaling parameters, $n$ is the number of updates, and $o_{0}$ is the prior action model which can be an incomplete action model or an empty action model (no predicates in preconditions and effects). Reliability is updated whenever $o$ is executed. The initial values of $S U$ and $V O$ are set to zero. It inherits the reliability of the prior model with $\beta \in(0,1)$ as the discount factor which reduces its significance given new data.

Success Rate. An action with a high success rate indicates that recent executions are successful which is more likely if it has a small error. We define the success rate as:

$$
\begin{aligned}
S U(o)= & \beta S U(o)+\mathbb{1}(\text { st }=\text { success }) \\
& +0.5 \times \mathbb{1}(\text { st }=\text { partial success })
\end{aligned}
$$

where $S U(o) \in\left[0, \frac{1}{1-\beta}\right)$, st is the execution status, and the indicator function $\mathbb{1}$ equals to 1 if the enclosing condition is 
true; otherwise, it is 0 . The execution status is 'failure' when the precondition of the action executed is not satisfied. The state is then assumed to be unchanged. The status is 'partial success' if the post-state is not expected given the learned effects. $S U$ is computed recursively with $\beta$ as the discount factor which gives less importance to past executions.

Volatility. Volatility measures how much a set of rules representing an action changes after learning. A low volatility suggests that learning has converged to the true action model. Volatility is computed recursively, and is defined as:

$$
V O(o)=\beta V O(o)+\tilde{d}\left(R_{\text {prev }}, R\right)
$$

where $V O(o) \in\left[0, \frac{1}{1-\beta}\right), R_{\text {prev }}(R)$ is the set of rules before (after) learning, and $\tilde{d}\left(R_{\text {prev }}, R\right)$ is the normalized difference between the two sets of rules. The difference between two rules is defined as:

$$
\begin{aligned}
d\left(r_{1}, r_{2}\right)= & d^{-}\left(r_{1}^{p}, r_{2}^{p}\right)+d^{-}\left(r_{2}^{p}, r_{1}^{p}\right) \\
& +d^{-}\left(r_{1}^{e}, r_{2}^{e}\right)+d^{-}\left(r_{2}^{e}, r_{1}^{e}\right)
\end{aligned}
$$

where superscripts $p$ and $e$ refer to the precondition and effect of a rule, respectively, and $d^{-}\left(p_{1}, p_{2}\right)$ returns the number of predicates that are in the set of predicates $p_{1}$ but not in $p_{2}$. The normalized difference is defined as:

$$
\tilde{d}\left(r_{1}, r_{2}\right)=\frac{d\left(r_{1}, r_{2}\right)}{\left|r_{1}\right|+\left|r_{2}\right|}
$$

where the operator $|r|$ refers to the number of predicates in $r$. The difference between two set of rules, $d\left(R_{1}, R_{2}\right)$, is the sum of differences of pairs of rules $r_{1} \in R_{1}$ and $r_{2} \in R_{2}$ where the rules are paired such that the sum is minimal. Each rule is paired at most once and the number of predicates in unpaired rules are added to the sum.

Exposure. Exposure measures the variability (inverse of similarity [Lang et al., 2012]) of the pre-states in the training data, and is defined as:

$$
E X(o)=\frac{N_{s}}{|\mathcal{S}| C_{2}} \sum_{s_{i}, s_{j} \in \mathcal{S}} \frac{d^{-}\left(s_{i}, s_{j}\right)}{\left|s_{i}\right|}+\frac{d^{-}\left(s_{j}, s_{i}\right)}{\left|s_{j}\right|}
$$

where $\mathcal{S}$ is the set of unique pre-states in the state transitions involving $o$, and $N_{s}$ is the number of state transitions resulting from successful executions. The first term is the ratio of state transitions from successful executions, penalizing those from failed executions which are less informative. Essentially, exposure is the average pairwise difference between pre-states weighted by $N_{s}$. Since probabilities of effects are inferred using maximum likelihood on successful state transitions, they converge to the true values as $N_{s}$ increases. Hence, reliability considers these probabilities implicitly.

\subsection{Model-Based Reinforcement Learning}

The inputs to the main ILM algorithm (Algorithm 1) are the prior action models $R_{0}$ and their reliability $R E_{0}$, initial state $s_{0}$, goal state $g$, planning horizon $H$, and the maximum number of iterations $N . E X_{\max }=0$ and $t a b u=\varnothing$ for the first episode. Exploration or exploitation is performed at the start

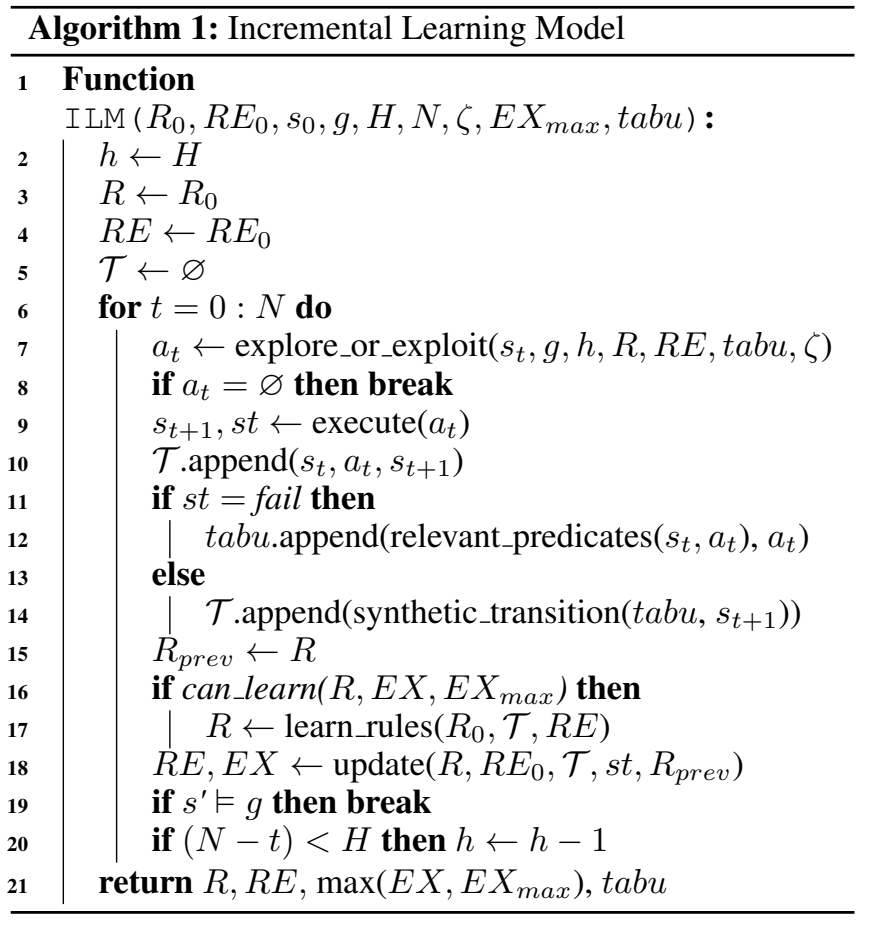

of each iteration (line 7). If no action is found, then a deadend is reached (line 8) and the algorithm terminates. When an action fails to execute, I LM learns from this failure by recording the failed instance in tabu (line 12: relevant_predicates returns the set of grounded predicates in $s$ that does not contain objects that were not in $a$ ), otherwise, synthetic state transitions $\left(s_{t}, a_{T}, s_{t}\right)$ are generated (line 14) where $a_{T}$ is a randomly grounded action such that check_tabu $\left(s_{t}, a_{T}, t a b u\right)$ $\Rightarrow \perp$. Failed executions are exceedingly less as failed instances are added to tabu. Reconstructing synthetic failed transitions augment the training data and aids the learning of rule preconditions.

Learning from training data of low variability (or low exposure) could result in existing rules being generalized where its literals that are true are removed in the updated rules preconditions or effects. To prevent this, we delay learning until certain criteria are met (can_learn in line 16):

1. If $R_{0}$ is the set of empty rules, always learn since no information can be lost. However, this risks learning incorrect preconditions or effects that can prevent the agent from reaching the goal state.

2. Otherwise, learn if there is at least one successful transition, at least one failed or synthetic transition, and $E X>\alpha_{E X} E X_{\max }$ where $\alpha_{E X} \in[0,1]$.

If learning is allowed, then new rules are learned (learn_rules in line 17) and the values of $R E, E X, V O$, and $S U$ are updated (line 18). Otherwise, only $R E, E X$, and $S U$ are updated. The algorithm terminates after reaching the maximum number of iterations or when the goal is reached. It returns the learned rules, reliability, maximum exposure $\left(E X_{\max }\right)$, and $t a b u$. These are used as inputs to the next function call to Algorithm 1. 


\section{Explore or Exploit}

We compute the counts for all applicable actions in $s$ using the context-based density formula from [Lang et al., 2012] which performs relational generalizations the amount of exploration is reduced as states which are unknown under propositional representations could be known under relational representations. The count-action pairs $\langle c, o\rangle$ are sorted in increasing order of $c=$ $R E(o) \sum_{r \in R} \sum_{\left(s, a, s^{\prime}\right) \in \mathcal{T}} \mathbb{1}(r$ is applicable in $s)$ in a list, $\mathcal{L}$, where $R$ are rules of $o$. Reliability serves as intrinsic motivation where less reliable actions are explored more.

A state is known if $\forall c_{i} \in \mathcal{L}\left(c_{i} \geq \zeta\right)$, or if the reliability of every action model exceeds a constant threshold. The second condition allows exploitation using prior action models when the counts are still zero. If the state is known, exploitation is attempted using Gourmand [Kolobov et al., 2012], a planner that solves problems modelled in finite-horizon MDPs online. (In general, ILM can use any planner that accepts planning problems written in PPDDL.) Exploitation fails if no plan is found or if the first action of the plan is in tabu.

Exploration is attempted if the state is not known or exploitation fails. An action is popped off the top of $\mathcal{L}$ and a list of grounded actions that are applicable in $s$ are enumerated. A grounded action that is not in tabu is selected at random and returned. If no such actions exist, then the next action is popped off until $\mathcal{L}$ is empty, following which random exploration is used where actions are grounded without considering whether preconditions are satisfied in $s$. If all grounded actions are in tabu, then a dead-end is reached.

\section{Learning from Failure}

Failed executions due to unsatisfied preconditions are recorded in tabu. Before an action $a$ is executed in state $s$, check_tabu(s,a,tabu) checks if $(s, a)$ is in tabu, returning False if so. We describe check_tabu with an example as shown in Figure 2. A state is described by a set of predicates. We extract the set of predicates $f_{s} \subseteq s$ that does not have an object in its binding that is not in the arguments of $a$. We assume that the arguments of actions are known. $f_{s}$ is compared to each entry $\left(f_{t}, a_{t}\right)$ in tabu. The predicates in $f_{t}$ are grounded with the same substitution as the variable bindings of $a$. Hence, the check is lifted to relational representations and is applicable even if the objects in the domain change. If $f_{s}$ does not have at least one predicate that is not in $f_{t}$, then $a$ is in tabu. In the example, moveCar $(l 31, l 13)$ is in tabu, as are all grounded actions of moveCar that do not have road(?loc1, ?loc2) in $f_{s}$. Intuitively, we know $s$ cannot satisfy the precondition of $a$ if it does not have any additional predicates as compared to a state where $a$ previously failed to execute. We exploit experiences from failed executions which are otherwise uninformative to the rules learner as it cannot determine the reason for the failure [Walsh et al., 2010].

Since every action is checked before execution, tabu will not contain identical entries. This keeps the size of tabu to a minimum which is important as the memory and time complexity is $\mathcal{O}(|t a b u|)$. The completeness of the algorithm depends on the failed instances in tabu. In the example, if tabu is $\varnothing$, then $a$ is not in tabu. It then fails to execute following which $f_{s}$ is lifted with $\sigma=\{l 31 \rightarrow$ ?loc $1, l 13 \rightarrow$ ?loc 2$\}$ and

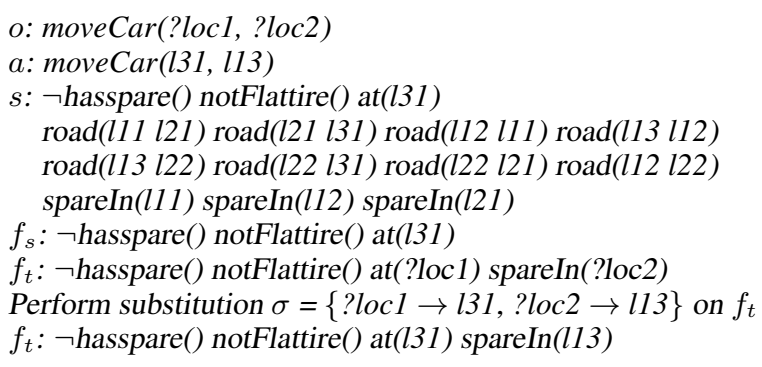

Figure 2: An example of checking if $(s, a)$ is in tabu.

inserted with $o$ in tabu. Since no erroneous instance is ever added, the algorithm is sound.

\subsection{Learning Action Models}

The rules learner from [Pasula et al., 2007] (called in line 17 of Algorithm 1) applies a search operator, selected at random, to a rule. Each search operator modifies the rule differently to yield a set of new rules. For example, a search operator adds a different predicate to the precondition or effect of a rule to generate a new rule. An example of a rule is shown in Figure 1. A greedy search uses a score function as a heuristic. We introduce a deviation penalty, $\operatorname{PEN}\left(R, R_{0}\right)$, to the score function such that the greedy search begins from and is bounded around the prior action models, $R_{0}$, which can be an empty rule, or rules of incomplete action models. Hence, the learner refines $R_{0}$. The score function is defined as:

$$
\begin{aligned}
\operatorname{Score}(R)= & \sum_{\left(s, a, s^{\prime}\right) \in \mathcal{T}} \log \left(\hat{P}\left(s^{\prime} \mid s, a, r_{(s, a)}\right)\right) \\
& -\alpha_{p} \sum_{r \in R} \operatorname{PEN}(r)-\operatorname{PEN}\left(R, R_{0}\right)
\end{aligned}
$$

where $\hat{P}$ is the probability of the effect in $r_{(s, a)}$ which covers the transition $\left(\mathrm{s}, \mathrm{a}, \mathrm{s}^{\prime}\right), \mathcal{T}$ is the training data, $\alpha_{p}$ is a parameter $\in[0,1]$, and $P E N(r)$ penalizes complex rules to avoid over-specialization. The deviation penalty increases when $R$ deviates further from $R_{0}$, and is defined as:

$$
\begin{array}{r}
\operatorname{PEN}\left(R, R_{0}\right)=\frac{R E\left(o_{0}\right)}{E X(o)}[ \\
\alpha_{\text {drop }} \Delta_{\text {drop }}\left(R, R_{0}\right)+ \\
\left.+\alpha_{a d d} \Delta_{a d d}\left(R, R_{0}\right)\right]
\end{array}
$$

where $\alpha_{d r o p}$ and $\alpha_{a d d}$ are scaling parameters $\in[0, \infty)$, and $\Delta_{d r o p}\left(R, R_{0}\right)$ and $\Delta_{a d d}\left(R, R_{0}\right)$ are defined as:

$$
\begin{aligned}
\Delta_{\text {drop }}\left(R, R_{0}\right) & =\frac{d^{-}\left(R_{0}^{p}, R^{p}\right)}{\left|R^{p}\right|+\left|R_{0}^{p}\right|}+\frac{d^{-}\left(R_{0}^{e}, R^{e}\right)}{\left|R^{e}\right|+\left|R_{0}^{e}\right|} \\
\Delta_{\text {add }}\left(R, R_{0}\right) & =\frac{d^{-}\left(R^{p}, R_{0}^{p}\right)}{\left|R^{p}\right|+\left|R_{0}^{p}\right|}
\end{aligned}
$$

where the pairings of rules $r \in R$ and $r_{0} \in R_{0}$ are the same as the pairings in $d\left(R, R_{0}\right)$.

Since past training data is not used, the rules learner may consider a probabilistic effect of $R_{0}$ as noise if this effect is rarely seen in the current training data. $\Delta_{d r o p}$ increases when 
this happens. If the probabilistic effect is not seen at all, it will be dropped in $R$ regardless of how large $\operatorname{PEN}\left(R, R_{0}\right)$ is. Such rules will be rejected. The deviation penalty is scaled by the reliability of the prior action model and the inverse of exposure. The intuition is that deviation should be limited if the prior action model is highly reliable, and encouraged if the training data has high variability.

\section{Experimental Results}

\subsection{Experimental Setup}

In one trial of experiments, ten planning problems are attempted sequentially in an order of increasing scale (see Table 1) following the idea behind curriculum learning [Bengio et al., 2009]. We denote each attempt as an episode. Each trial starts with no prior knowledge; the prior action models for episode 1 are empty action models. Since the planning problems are probabilistic, 50 independent trials are conducted. The machine used to run the experiments was a four core Intel(R) i5-6500 with 4 GB of RAM.

We used three planning domains: the Tireworld and Exploding Blocksworld domains from the International Probabilistic Planning Competition [Younes et al., 2005], and the Logistics domain. In the Tireworld domain, the car may get a flat tire when moving to another location. If the tire is flat, the car cannot move and a dead-end is reached if no spare tires are available. Tireworld problems of the same scale are identical and are constructed systematically such that there are no unavoidable dead-ends [Little and Thiebaux, 2007]. In the Exploding Blocksworld domain, a block may detonate when it is put down, destroying the block or table beneath. A destroyed block or table is no longer accessible. Each block can only detonate once. We set the goal states as random configurations of three blocks. All Logistics problems have one truck per city, one airplane, and one parcel. Loading and unloading parcels may fail and the state remains unchanged. The models for all domains are stationary where probabilities of the effects of actions are kept constant in all episodes.

The performance of ILM is evaluated with the correctness of the learned model and the goal-directedness. R-MAX and two variants of ILM are included for comparison. ILM-R does not use reliability; the relational count is not weighted and the deviation penalty in the score function used by the rules learner is zero. It does not delay learning (line 15 of Algorithm 1) as this requires $E X_{\max }$, a component of reliability. ILM-T does not learn from failure. ILM, ILM-R, and ILM- $\mathrm{T}$ do not use past training data while $\mathrm{R}-\mathrm{MAX}$ does.

\subsection{Correctness of Learned Models}

The correctness of a learned model $\hat{P}$ can be defined as the average variational distance between $\hat{P}$ and the true model $P$ [Pasula et al., 2007]:

$$
V D(P, \hat{P})=\frac{1}{|\mathcal{T}|} \sum_{T_{i} \in \mathcal{T}}\left|P\left(T_{i}\right)-\hat{P}\left(T_{i}\right)\right|
$$

where $\mathcal{T}$ is the set of test examples - 500 state transitions per action are generated with the true distribution. Figure 3 show the variational distances for Tireworld,

\begin{tabular}{ccccc}
\hline \hline Scale & Episode & Tireworld & $\begin{array}{c}\text { Exploding } \\
\text { Blocksworld }\end{array}$ & Logistics \\
\hline Small & 1 to 3 & 6 locations & 5 blocks & $\begin{array}{c}2 \text { cities, } \\
4 \text { locations } \\
2 \text { cities, }\end{array}$ \\
Medium & 4 to 6 & 15 locations & 7 blocks & $\begin{array}{c}6 \text { locations } \\
3 \text { cities, }\end{array}$ \\
Large & 7 to 10 & 28 locations & 9 blocks & 8 locations \\
\hline
\end{tabular}

Table 1: Number of objects in small, medium, and large-scale planning problems for each of the three domains.

Exploding Blocksword, and Logistics. The variational distances at episode 0 are of the prior action models, which are empty models, for episode 1 .

Tireworld. ILM learns action models incrementally as evident by the decrease in variational distance from episodes 1 to 10. ILM-R performed marginally worse as it learns from training data of low variability which caused the variational distances to increase in episodes 4 and 6 . The utility of learning from failure is illustrated by the significantly larger variational distances for ILM-T and R-MAX. In both cases, most of the executions led to failure which are less meaningful experiences for the rules learner. Since the maximum number of iterations is only 15 (moveCar alone has 36 possible groundings for the small-scale planning problems), such inefficient exploration performs poorly.

Exploding Blocksworld. The lowest variational distances are achieved with I LM from episodes 1 to 4 and with R-MAX thereafter. The latter learns from a larger training set which is important for this domain which has complex actions putOnBlock and putDown. These actions have conditional effects which are modelled as separate rules with different preconditions. Preconditions are inferred by comparing pre-states in the training data. Most of the predicates in the pre-states remain unchanged as an action typically changes a small subset of the state. Hence, more training data is required to learn more complex preconditions. Since the training data used by $\mathrm{R}-\mathrm{MAX}$ are largely from failed experiences, it took four episodes before it outperforms ILM.

Logistics. ILM had the best performance in all episodes. The large variational distances for ILM- $\mathrm{T}$ is due to the difficulty in learning driveTruck. This action has four arguments and has 432 possible groundings in the small-scale planning problems. This has complications in the goal-directedness which shall be discussed in the next subsection.

\subsection{Goal-directedness}

We evaluate the goal-directedness by the number of successful trials which are trials of an episode where the goal state is reached. The goal-directedness for the three domains is shown in Table 2. It is averaged over episodes with planning problems of the same scale. Episode 1 is separated from episodes 2 and 3 to illustrate the advantage of having prior knowledge. The average number of successful trials for episodes 2 and 3 were generally larger than episode 1 even though the scales of the planning problems are the same. This 

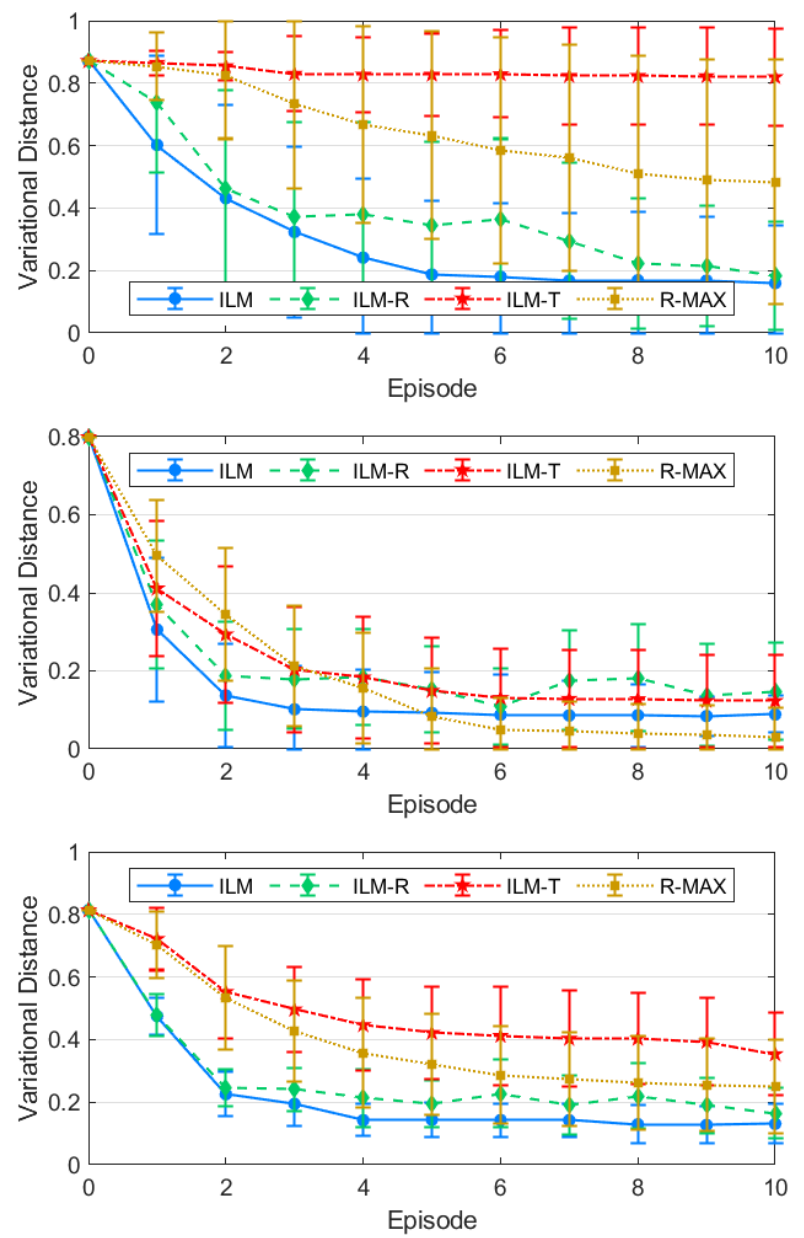

Figure 3: Variational distance at the end of each episode for, from top to bottom, the Tireworld, Exploding Blocksworld, and Logistics domains. The results are the means and standard deviations of 50 trials.

is because ILM exploits the learned models from the previous episode whereas episode 1 had no such prior knowledge.

Tireworld. ILM-R outperforms ILM in episodes 1 to 3 as the goal state can be reached by executing moveCar repeatedly as long as the tire is not flat along the way. I LM attempts exploitation more often than ILM-R as it weights relational counts with reliability. For small-scale planning problems, exploration or exploitation may not make a significant difference. When the scale increases, the number of steps between the initial state and the goal state increases and the probability of getting a flat tire along the way is higher. A dead-end is reached if the tire is flat and no spare tire is available. In such circumstances, exploitation is required and ILM outperforms I LM-R in episodes 4 to 10. ILM-T and R-MAX did not perform well as actions failed to execute most of the time.

Exploding Blocksworld. Dead-ends are often the cause of failing to reach the goal state. A block could detonate with a probability of 0.2 when executing putDown or putOnBlock which destroys the table or the underlying block. These irre-

\begin{tabular}{r|rrrrrrrrrrrr}
\hline \multirow{2}{*}{ Episode } & \multicolumn{3}{|c}{ ILM } & \multicolumn{1}{c}{ ILM-R } & \multicolumn{3}{c}{ ILM-T } & \multicolumn{2}{c}{ R-MAX } \\
\cline { 2 - 13 } & T & E & L & T & E & L & T & E & L & T & E & L \\
\hline 1 & 18 & 4 & 2 & 22 & 1 & 3 & 3 & 0 & 0 & 6 & 0 & 0 \\
2 to 3 & 38 & 22 & 10 & 42 & 10 & 5 & 6 & 4 & 0 & 16 & 10 & 3 \\
4 to 6 & 17 & 27 & 20 & 16 & 12 & 9 & 2 & 9 & 3 & 13 & 8 & 6 \\
7 to 10 & 6 & 17 & 18 & 4 & 7 & 16 & 1 & 9 & 9 & 2 & 9 & 17 \\
\hline
\end{tabular}

Table 2: Average number of successful trials for Tireworld (T), Exploding Blocksworld(E), and Logistics (L) domains.

versible changes could then lead to dead-ends. ILM has the most number of successful trials in all episodes. ILM-R performed much poorer than ILM as reaching the goal state with exploration alone is difficult. Even though R-MAX has lower variational distances than ILM for episodes 5 to 10 , it did not outperform ILM as it does not learn from failure.

Logistics. The number of successful trials increases even when the scale of the planning problem increases. In smallscale planning problems, there were few successful trials because driveTruck was not learned yet as mentioned previously. driveTruck failed to execute repeatedly until episode 3 as only two out of 432 grounded actions would succeed. As a result, a subset of the state space, which could include the goal state, is not reached. If states where the truck is at a location with a parcel are never reached, then loadTruck and unloadTruck could not be executed. This applies to loadAirplane and unloadAirplane if a parcel is not at an airport.

\section{Conclusions and Future Work}

We presented an MBRL approach called ILM which learns action models incrementally over episodes without the use of past training data. We introduced a new measure, reliability, which serves as an empirical estimate of the learning progress and influences the processes of learning and planning. We also extended an existing rules learner to consider prior knowledge in the form of incomplete action models. ILM learns from failure and avoids repeating similar failures, thereby improving the learning efficiency. We evaluated I LM on three benchmark domains. Experimental results showed that variational distances of learned action models decreased over each subsequent episode. Learning from failure greatly reduced the number of failed executions leading to improved correctness and goal-directedness.

For complex domains, more training data is required to learn action models. While we could use past training data, this would not work well for non-stationary domains and also increases the computation time for learning. The first issue could be resolved by learning the distributions from the current training data only. The second issue could be resolved by maintaining a fixed size of training data by replacing older experiences while maximizing the exposure, or variability, of the training data. These will be explored in the future.

\section{Acknowledgements}

This work was partially funded by the EPSRC ORCA Hub under grant number EP/R026173/1. 


\section{References}

[Bengio et al., 2009] Yoshua Bengio, Jérôme Louradour, Ronan Collobert, and Jason Weston. Curriculum learning. In Proc. ICML, pages 41-48, 2009.

[Brafman and Tennenholtz, 2002] Ronen I Brafman and Moshe Tennenholtz. R-MAX - A general polynomial time algorithm for near-optimal reinforcement learning. JMLR, 3:213-231, 2002.

[Chentanez et al., 2005] Nuttapong Chentanez, Andrew G Barto, and Satinder P Singh. Intrinsically motivated reinforcement learning. In Proc. NIPS, pages 1281-1288, 2005.

[Cullen and Bryman, 1988] Jet Cullen and Alan Bryman. The knowledge acquisition bottleneck: time for reassessment? Expert Systems, 5(3):216-225, 1988.

[Diuk et al., 2009] Carlos Diuk, Lihong Li, and Bethany R Leffler. The adaptive k-meteorologists problem and its application to structure learning and feature selection in reinforcement learning. In Proc. ICML, pages 249-256, 2009.

[Gil, 1994] Yolanda Gil. Learning by experimentation: Incremental refinement of incomplete planning domains. In Proc. ICML, pages 87-95, 1994.

[Guestrin et al., 2002] Carlos Guestrin, Relu Patrascu, and Dale Schuurmans. Algorithm-directed exploration for model-based reinforcement learning in factored mdps. In Proc. ICML, pages 235-242, 2002.

[Hester and Stone, 2017] Todd Hester and Peter Stone. Intrinsically motivated model learning for developing curious robots. AIJ, 247:170-186, 2017.

[Kearns and Koller, 1999] Michael Kearns and Daphne Koller. Efficient reinforcement learning in factored MDPs. In Proc. IJCAI, pages 740-747, 1999.

[Kolobov et al., 2012] Andrey Kolobov, Mausam, and Daniel S Weld. LRTDP vs. UCT for online probabilistic planning. In Proc. AAAI, page 1786-1792, 2012.

[Lang et al., 2012] Tobias Lang, Marc Toussaint, and Kristian Kersting. Exploration in relational domains for modelbased reinforcement learning. JMLR, 13:3725-3768, 2012.

[Little and Thiebaux, 2007] Iain Little and Sylvie Thiebaux. Probabilistic planning vs. replanning. In Proc. ICAPS Workshop on the International Planning Competition: Past, Present and Future, 2007.

[Martínez et al., 2016] David Martínez, Guillem Alenya, Carme Torras, Tony Ribeiro, and Katsumi Inoue. Learning relational dynamics of stochastic domains for planning. In Proc. ICAPS, page 235-243, 2016.

[Mourão et al., 2012] Kira Mourão, Luke S Zettlemoyer, Ronald P. A. Petrick, and Mark Steedman. Learning STRIPS operators from noisy and incomplete observations. In Proc. UAI, pages 614-623, 2012.

[Oates and Cohen, 1996] Tim Oates and Paul R Cohen. Learning planning operators with conditional and probabilistic effects. In Proc. AAAI Spring Symposium on Plan- ning with Incomplete Information for Robot Problems, pages 86-94, 1996.

[Pasula et al., 2007] Hanna M Pasula, Luke S Zettlemoyer, and Leslie Pack Kaelbling. Learning symbolic models of stochastic domains. JAIR, 29:309-352, 2007.

[Rodrigues et al., 2010] Christophe Rodrigues, Pierre Gérard, and Céline Rouveirol. Incremental learning of relational action models in noisy environments. In Proc. ILP, pages 206-213, 2010.

[Walsh et al., 2010] Thomas J Walsh, Kaushik Subramanian, Michael L Littman, and Carlos Diuk. Generalizing apprenticeship learning across hypothesis classes. In Proc. ICML, pages 1119-1126, 2010.

[Wang, 1995] Xuemei Wang. Learning by observation and practice: An incremental approach for planning operator acquisition. In Proc. ICML, pages 549-557, 1995.

[Younes and Littman, 2004] Håkan LS Younes and Michael L Littman. PPDDL1. 0: An extension to PDDL for expressing planning domains with probabilistic effects. Technical Report CMU-CS-04-162, 2004.

[Younes et al., 2005] Håkan LS Younes, Michael L Littman, David Weissman, and John Asmuth. The first probabilistic track of the International Planning Competition. JAIR, 24:851-887, 2005.

[Zhuo et al., 2013] Hankz Hankui Zhuo, Tuan Anh Nguyen, and Subbarao Kambhampati. Refining incomplete planning domain models through plan traces. In Proc. IJCAI, pages 2451-2458, 2013. 